Prons

trobertivier

Journal of Nonlinear Mathematical Physics

ISSN (Online): 1776-0852 ISSN (Print): 1402-9251

$P^{\text {rrumisis }}$ Journal Home Page: https://www.atlantis-press.com/journals/jnmp

\title{
Darboux transformation and novel solutions for the long wave-short
} wave model

Xin Huang, Boling Guo, Liming Ling

To cite this article: Xin Huang, Boling Guo, Liming Ling (2013) Darboux transformation and novel solutions for the long wave-short wave model, Journal of Nonlinear Mathematical Physics 20:4, 514-528, DOI:

https://doi.org/10.1080/14029251.2013.868265

To link to this article: https://doi.org/10.1080/14029251.2013.868265

Published online: 04 January 2021 


\title{
Darboux transformation and novel solutions for the long wave-short wave model
}

\author{
Xin Huang \\ College of Mathematics and Software Science, Sichuan Normal University, Chengdu 610066, Sichuan, China \\ Department of Basic Courses, Sichuan Finance and Economics Vocational College, Chengdu 610101, Sichuan, China \\ Boling Guo \\ Institute of Applied Physics and Computational Mathematics, Beijing 100088, China \\ Liming Ling* \\ School of Sciences, South China University of Technology, Guangzhou 510640, China \\ linglm@scut.edu.cn,lingliming@qq.com
}

Received 17 June 2013

Accepted 25 September 2013

\begin{abstract}
Firstly, we establish the relation between the loop group method and gauge transformation method for $3 \times 3$ spectral problem. Some novel solutions of long wave-short wave model are obtained by Darboux transformation method. Besides, we give the analysis and classification of solution in detail.

Keywords: Long wave-short wave, Soliton, Exact solution, Darboux transformation, Non-vanishing background.
\end{abstract}

2000 Mathematics Subject Classification: 37K10, 37K35, 37K40

\section{Introduction}

In 1978, Newell presented a long wave-short wave model [17]

$$
\begin{aligned}
A_{t} & =2 \sigma K_{1}\left(|B|^{2}\right)_{x}, \quad \sigma= \pm 1, \\
B_{t}-\mathrm{i} K_{2} B_{x x} & =-K_{3} A_{x} B+\mathrm{i} K_{4} A^{2} B-2 \mathrm{i} \sigma K_{5} B|B|^{2},
\end{aligned}
$$

where $A(x, t)$ and $B(x, t)$ represent the amplitude of the long wave and the envelope of the short wave respectively. The system (1.1) is integrable for the choices of parameters $K_{i}=1, i=1,2,3,4,5$ [17]. Another relative long wave-short wave model was derived by Benney in 1977 [2], which had been widely researched, such as $[8,12,15,16,19]$. However, there is few research about the model $(1.1)$.

In this work, we consider the long wave-short wave model (1.1) with the choices $K_{i}=1$ and $\sigma=1$

$$
\begin{array}{r}
\mathrm{i} B_{t}+B_{x x}+\left(\mathrm{i} A_{x}+A^{2}\right) B+2|B|^{2} B=0, \\
A_{t}+2\left(|B|^{2}\right)_{x}=0 .
\end{array}
$$

$\overline{{ }^{*} \text { Corresponding Author. }}$ 
It is readily to see that the equations (1.2) possess the scalings invariant property $x \rightarrow \lambda x, t \rightarrow \lambda^{2} t$, $A \rightarrow A / \lambda$ and $B \rightarrow B / \lambda$. Owning to the integrability of the long wave-short wave model (1.2), we can rewritten it as the compatibility condition of the following linear system [17]

$$
\begin{aligned}
& \Phi_{x}=U \Phi, \\
& \Phi_{t}=V \Phi,
\end{aligned}
$$

where

$$
\begin{aligned}
& U=\left[\begin{array}{ccc}
\mathrm{i} \lambda & B & \mathrm{i} A \\
-B^{*} & 0 & -B^{*} \\
\mathrm{i} A & B & -\mathrm{i} \lambda
\end{array}\right], \\
& V=\left[\begin{array}{ccc}
-\frac{\mathrm{i}}{3} \lambda^{2}+\mathrm{i}|B|^{2} & -B \lambda+\mathrm{i} B_{x}-A B & -\mathrm{i}|B|^{2} \\
B^{*} \lambda+\mathrm{i} B_{x}^{*}+A B^{*} & \frac{2 \mathrm{i}}{3} \lambda^{2}-2 \mathrm{i}|B|^{2} & -B^{*} \lambda+\mathrm{i} B_{x}^{*}+A B^{*} \\
-\mathrm{i}|B|^{2} & B \lambda+\mathrm{i} B_{x}-A B & -\frac{\mathrm{i}}{3} \lambda^{2}+\mathrm{i}|B|^{2}
\end{array}\right] .
\end{aligned}
$$

From above spectral problem (1.3), we can see that it is nothing but deep reduction of three wave resonance interaction(3WRI) [13,20]. Also, the $3 \mathrm{WRI}$ model is well researched by lots of researchers, such as $[3,4,6,13,20]$. Soliton solutions of $3 \mathrm{WRI}$ can be classified in three main classes, depending on the boundary conditions. The first one is the bright-bright-bright solitons [13,20], where the pulses of the three interacting waves vanish sufficiently fast at infinity. The second class, brightbright-dark solitons, is given by two bright pulses which vanish sufficiently fast at infinity and a kink-like pulse which asymptotically behaves like a plane wave [3]. The third class, dark-dark-dark solitons, is given in [4]. The detailed analysis of the $3 \mathrm{WRI}$ spectral problem presented in reference [6].

As we well known that, the Darboux transformation method [7,16] is an efficient way to generate the soliton solution. In paper [5], the author establishes the relations between different construction methods. However, the classical Darboux transformation can not tackle with the high-order spectral. Recently, Guo, Ling and Liu $[9,10]$ develop the Darboux transformation, so that it can be used to yield the high order solution. Afterwards, Bian, Guo and Ling combine the generalized Darboux transformation with the inverse scattering method, so that the inverse scattering method can be used to generate the high-order solution readily [1].

In reference [14], the authors give the Darboux transformation for about linear system (1.3) and present some simple exact solutions. In reference [11], the authors give a method to solve the Lax pair with the nonzero seed solution. In this paper, we combine the method in [11] with [14] to construct some novel solutions of equations (1.2).

This paper is organized as following. In section 2, we give the Darboux transformation by the loop group method. We prove the equivalence between the loop group representation and gauge transformation representation. In section 3, we give some novel solutions and classification of solution. We analyse the asymptotical behavior of the new types soliton solution in detail. Final section involves some conclusions and discussions.

\section{Darboux transformation}

We use loop group method [18] to derive the Darboux transformation. Since the spectral problem (1.3) is the $3 \times 3$ AKNS spectral problem, then the elementary Darboux transformation of (1.3) can 
be represented as the following form [18]:

$$
T=I+\frac{P}{\lambda-\lambda_{1}^{*}}
$$

where $P$ is the projector operator. On the other hand, we introduce the involution relation for linear system (1.3). It is evidently that the matrices $U(\lambda)$ and $V(\lambda)$ possess the following involution relations

$$
U^{\dagger}\left(\lambda^{*}\right)=-U(\lambda), \quad V^{\dagger}\left(\lambda^{*}\right)=-V(\lambda)
$$

and

$$
\sigma_{2} U(\lambda) \sigma_{2}=U(-\lambda), \quad \sigma_{2} V(\lambda) \sigma_{2}=V(-\lambda)
$$

where

$$
\sigma_{2}=\left[\begin{array}{lll}
0 & 0 & 1 \\
0 & 1 & 0 \\
1 & 0 & 0
\end{array}\right] .
$$

Based on the above involution relations, we establish the following lemma;

Lemma 2.1. If $U$ and $V$ satisfy the involution relations (2.1) and (2.2), then there exists a fundamental solution $\Phi(\lambda)$ for linear system (1.3) which satisfies the symmetry relations $\Phi^{\dagger}\left(\lambda^{*}\right) \Phi(\lambda)=I$ and $\Phi(-\lambda)=\sigma_{2} \Phi(\lambda) \sigma_{2}$; and vice versa.

Proof: Since the symmetry relation (2.1), we can deduce that the fundamental solution $\Phi^{\dagger}\left(\lambda^{*}\right)$ satisfies the following conjugation equation

$$
\begin{aligned}
& -\Phi_{x}^{\dagger}\left(\lambda^{*}\right)=\Phi^{\dagger}\left(\lambda^{*}\right) U(\lambda), \\
& -\Phi_{t}^{\dagger}\left(\lambda^{*}\right)=\Phi^{\dagger}\left(\lambda^{*}\right) V(\lambda) .
\end{aligned}
$$

On the other hand, the solution $\Phi^{-1}(\lambda)$ satisfy the above conjugation linear system. Moreover, we have $\Phi(\lambda) \Phi^{\dagger}\left(\lambda^{*}\right)=C_{1}$. Specially, we can choose the constant matrix $C_{1}=I$. Similarly, by relation (2.2), we can obtain the matrix $\sigma_{2} \Phi(\lambda) \sigma_{2}$ satisfies the following system

$$
\begin{aligned}
& \sigma_{2} \Phi_{x}(\lambda) \sigma_{2}=U(-\lambda) \sigma_{2} \Phi(\lambda) \sigma_{2}, \\
& \sigma_{2} \Phi_{t}(\lambda) \sigma_{2}=V(-\lambda) \sigma_{2} \Phi(\lambda) \sigma_{2} .
\end{aligned}
$$

Then we have $\Phi(-\lambda)=\sigma_{2} \Phi(\lambda) \sigma_{2} C_{2}$. In particular, we choose the matrix $C_{2}=I$. By the above analysis, converse process is evidently valid.

By above lemma, we can obtain the symmetry relation for Darboux matrix $T$, i.e. $T(x, t ;-\lambda)=$ $\sigma_{2} T(x, t ; \lambda) \sigma_{2}$ and $T^{\dagger}\left(x, t ; \lambda^{*}\right) T(x, t ; \lambda)=I$. Furthermore, we can suppose

$$
T=I+\frac{A_{1}}{\lambda-\lambda_{1}^{*}}-\frac{\sigma_{2} A_{1} \sigma_{2}}{\lambda+\lambda_{1}^{*}}, \quad T^{-1}=I+\frac{A_{1}^{\dagger}}{\lambda-\lambda_{1}}-\frac{\sigma_{2} A_{1}^{\dagger} \sigma_{2}}{\lambda+\lambda_{1}} .
$$

The matrix $A_{1}$ has two distinct cases. One case is the rank of matrix $A_{1}$ equals to one. Another case is the rank of matrix $A_{1}$ equals to two. Although its possess different rank, its are equivalence each other actually. In what following, we would like to verify this fact. 
Prior to prove the fact, we give the following lemma. The lemma establishes the relation between loop group method and gauge transformation method.

\section{Lemma 2.2. The Darboux matrix}

$$
T=I+\frac{\lambda_{1}^{*}-\lambda_{1}}{\lambda-\lambda_{1}^{*}} P, \quad P \text { is a projector matrix }
$$

can be rewritten as

$$
T=M\left[\begin{array}{ccc}
\frac{\lambda-\lambda_{1}}{\lambda-\lambda_{1}^{*}} & 0 & 0 \\
0 & 1 & 0 \\
0 & 0 & 1
\end{array}\right] M^{-1}, \quad M=\left[\Phi_{1}\left(\lambda_{1}\right), \Phi_{2}\left(\lambda_{1}^{*}\right), \Phi_{3}\left(\lambda_{1}^{*}\right)\right],
$$

or

$$
T=N\left[\begin{array}{ccc}
\frac{\lambda-\lambda_{1}}{\lambda-\lambda_{1}^{*}} & 0 & 0 \\
0 & \frac{\lambda-\lambda_{1}}{\lambda-\lambda_{1}^{*}} & 0 \\
0 & 0 & 1
\end{array}\right] N^{-1}, \quad N=\left[\Phi_{1}\left(\lambda_{1}\right), \Phi_{2}\left(\lambda_{1}\right), \Phi_{3}\left(\lambda_{1}^{*}\right)\right]
$$

Proof: Assume the rank of $P$ equals to one, and the kernel of matrix $I-P$ is $\Phi_{1}$. By the relation $\Phi^{\dagger}\left(\lambda^{*}\right) \Phi(\lambda)=I$, we can deduce that the $\Phi_{2}\left(\lambda_{1}^{*}\right)$ and $\Phi_{3}\left(\lambda_{1}^{*}\right)$ are the kernel of $P$. Therefore, we can obtain that the

$$
P=M\left[\begin{array}{lll}
1 & 0 & 0 \\
0 & 0 & 0 \\
0 & 0 & 0
\end{array}\right] M^{-1}
$$

It follows that the equation (2.4) is valid. Similarly, if the rank of $P$ equals to two, the equation (2.5) is valid.

Theorem 2.1. The Darboux matrix (2.5) can be rewritten as the following form

$$
T=\frac{\lambda-\lambda_{1}^{*}}{\lambda-\lambda_{1}}\left(I+\frac{\lambda_{1}-\lambda_{1}^{*}}{\lambda-\lambda_{1}} P_{1}\right)
$$

where

$$
P_{1}=\frac{\Phi_{3}\left(\lambda_{1}^{*}\right) \Phi_{3}^{\dagger}\left(\lambda_{1}^{*}\right)}{\Phi_{3}^{\dagger}\left(\lambda_{1}^{*}\right) \Phi_{3}\left(\lambda_{1}^{*}\right)}
$$

Proof: The directly calculating.

By above theorem, we know that the rank of matrix $P_{1}$ equals to one. Thus for the $3 \times 3$ spectral problem, we merely need to choose the rank of matrix $P$ equals to one. It follows that the rank of matrix $A_{1}$ equals to one, since the form of Darboux matrix (2.3) is nothing but iteration of elementary Darboux transformation twice. Then we can suppose the matrix $A_{1}=\left|y_{1}\right\rangle\left\langle x_{1}\right|$, where $\left|y_{1}\right\rangle$ is a row vector, $\left\langle x_{1}\right|$ is a column vector, and $\left.\left|x_{1}\right\rangle=\left\langle\left. x_{1}\right|^{\dagger},\right\rangle y_{1}|=| y_{1}\right\rangle^{\dagger}$. By the relation $T(\lambda) T^{\dagger}\left(\lambda^{*}\right)=I$, 
we have the following residue relation

$$
\operatorname{Res}_{\lambda=\lambda_{1}^{*}}\left(T(\lambda) T^{\dagger}\left(\lambda^{*}\right)\right)=0
$$

Then we can have

$$
\left(I+\frac{\left|y_{1}\right\rangle\left\langle x_{1}\right|}{\lambda_{1}-\lambda_{1}^{*}}-\frac{\sigma_{2}\left|y_{1}\right\rangle\left\langle x_{1}\right| \sigma_{2}}{\lambda_{1}+\lambda_{1}^{*}}\right)\left|y_{1}\right\rangle=0
$$

We can obtain two messages from above equation. Firstly, we can suppose that $\left|x_{1}\right\rangle=\Phi_{1}\left(\lambda_{1}\right)$, where $\Phi_{1}\left(\lambda_{1}\right)$ is a special solution for linear system (1.3) with $\lambda=\lambda_{1}$. Secondly, we can solve the $\left|y_{1}\right\rangle$ by above linear equations. Denote

$$
\alpha=\frac{\left\langle x_{1}\left|\sigma_{2}\right| x_{1}\right\rangle}{\lambda_{1}+\lambda_{1}^{*}}, \quad \beta=\frac{\left\langle x_{1} \mid x_{1}\right\rangle}{\lambda_{1}-\lambda_{1}^{*}}
$$

It follows that

$$
\left|y_{1}\right\rangle=\frac{1}{\alpha^{2}-\beta^{2}}\left(\alpha \sigma_{2}+\beta\right)\left|x_{1}\right\rangle .
$$

Because the Darboux transformation keep the matrix form, then we have the following relation $T_{x}+T U=\widehat{U} T$. Taking the spectral parameter large enough $\lambda \rightarrow \infty$, we have the transformation between the fields, i.e.

$$
\begin{aligned}
& A[1]=A-\frac{2}{\alpha^{2}-\beta^{2}}\left[\beta\left(\phi_{1} \phi_{3}^{*}-\phi_{3} \phi_{1}^{*}\right)+\alpha\left(\left|\phi_{3}\right|^{2}-\left|\phi_{1}\right|^{2}\right)\right], \\
& B[1]=B-\mathrm{i} \frac{\left(\phi_{3}-\phi_{1}\right) \phi_{2}^{*}}{\alpha+\beta}
\end{aligned}
$$

where $\phi_{i}$ equals to the $i$-th component of $\left|x_{1}\right\rangle$. The $\mathrm{N}$-fold Darboux transformation is given in reference [14], we omit it in this work.

\section{Exact solution with non-vanishing background}

In reference [14], the authors choose two special seed solutions. In this paper, we consider the general seed solution. It is readily to obtain the seed solution

$$
A=d, \quad B=c \exp \left[\mathrm{i}\left(a x+\left(d^{2}+2 c^{2}-a^{2}\right) t\right)\right]
$$

readily, where $a, b, c$ and $d$ are real constants. In what following, we use the method in reference [11] to solve the linear system (1.3) with seed solution (3.1). Although we can solve the linear system with a unified formula, there are two cases to simple formulas. Thus we classify three different cases to construct the fundamental solution.

\subsection{The case I}

In this case, we consider the special case in (3.1) with $c=0$ and $d \neq 0$. Under scaling transformation, we merely need to consider $d=1$. In order to avoid the radical sign, we use a simple substitution $\lambda_{1}=\frac{1}{2}\left(k-\frac{1}{k}\right)$. We can obtain a general vector solution for linear system (1.3). We would like to 
give the detailed calculating in subsection 3.3. In this subsection, we merely give the results. We can obtain that

$$
\left|x_{1}\right\rangle=\left[\begin{array}{c}
\exp \left[-\frac{\mathrm{i}}{12}\left(k-k^{-1}\right)^{2} t\right]\left(c_{1} \exp \left[\frac{\mathrm{i}}{2}\left(k+k^{-1}\right) x\right]+c_{2} \exp \left[-\frac{\mathrm{i}}{2}\left(k+k^{-1}\right) x\right]\right) \\
c_{3} \exp \left[\frac{\mathrm{i}}{6}\left(k-k^{-1}\right)^{2} t\right] \\
\exp \left[-\frac{\mathrm{i}}{12}\left(k-k^{-1}\right)^{2} t\right]\left(c_{1} k^{-1} \exp \left[\frac{\mathrm{i}}{2}\left(k+k^{-1}\right) x\right]-c_{2} k \exp \left[-\frac{\mathrm{i}}{2}\left(k+k^{-1}\right) x\right]\right)
\end{array}\right],
$$

where $c_{1}$ and $c_{2}$ are complex constants. For convenience, we choose the parameter $c_{3}=1$ and divide $\left|x_{1}\right\rangle$ by the factor $\exp \left[\frac{i}{6}\left(k-k^{-1}\right)^{2} t\right]$, then we can obtain

$$
\left|x_{1}\right\rangle=\left[\begin{array}{c}
\exp \left[-\frac{\mathrm{i}}{4}\left(k-k^{-1}\right)^{2} t\right]\left(c_{1} \exp \left[\frac{\mathrm{i}}{2}\left(k+k^{-1}\right) x\right]+c_{2} \exp \left[-\frac{\mathrm{i}}{2}\left(k+k^{-1}\right) x\right]\right) \\
1 \\
\exp \left[-\frac{\mathrm{i}}{4}\left(k-k^{-1}\right)^{2} t\right]\left(c_{1} k^{-1} \exp \left[\frac{\mathrm{i}}{2}\left(k+k^{-1}\right) x\right]-c_{2} k \exp \left[-\frac{\mathrm{i}}{2}\left(k+k^{-1}\right) x\right]\right)
\end{array}\right] .
$$

Together with equations (2.6), (3.1) and (3.2), we can obtain the exact solutions. Because the exact solution formulas are rather complex, we omit the explicit expression. We can obtain three kinds of nontrivial solution by formulas (2.6).

\subsubsection{Bright-Dark pair}

If we choose the parameters $c_{1}=0$ and $c_{2} \neq 0, k=e+\mathrm{i} f, e f \neq 0$ and $e>0$, we can obtain the exact expression for $A$ and $B$ :

$$
\begin{aligned}
& A=1-\frac{\gamma}{\delta\left[e^{2}(\omega+1)^{2} e^{2 X_{1}}+\omega e^{-2 X_{1}}\right]+1}, \\
& B=\frac{e f\left(\omega^{2}-1\right) e^{\mathrm{i} Y_{1}}}{\omega\left[e(\omega+1)(1-e+\mathrm{i} f) e^{X_{1}}-(\omega-e+\mathrm{i} f) e^{-X_{1}}\right]},
\end{aligned}
$$

where

$$
\begin{aligned}
X_{1} & =\frac{f}{2}\left(1-\frac{1}{\omega}\right)\left(x+\left(e+\frac{e}{\omega}\right) t+g_{1}\right), \quad \omega=e^{2}+f^{2}, \\
Y_{1} & =-\frac{e}{2}\left(1+\frac{1}{\omega}\right)\left(x+\left(\frac{e}{2}+\frac{e}{\omega}-\frac{f^{2}+1}{2 e}-\frac{2 e}{\omega+1}\right) t+g_{2}\right), \\
\gamma & =\frac{(\omega-1)^{2} f^{2}}{\left[e\left((e-1)^{2}+f^{2}\right)-2 f^{2}\right] \omega}, \quad g_{1}=\frac{2 \omega \ln \left|c_{2}\right|}{f(\omega-1)}, \\
\delta & =\frac{\left((e-1)^{2}+f^{2}\right)}{2 e(\omega+1)\left(e\left((e-1)^{2}+f^{2}\right)-2 f^{2}\right)}, \quad g_{2}=\frac{-2 \omega \arg \left(c_{2}\right)}{e(1+\omega)} .
\end{aligned}
$$

The trajectories of $A$ and $|B|^{2}$ are along the line $X_{1}+\ln (|e|(1+\omega))-\frac{1}{2} \ln (\omega)=0$. The cavity depth for $A$ is

$$
A_{c d}=\frac{(\omega-1)^{2} f^{2}}{\omega\left[\left(e+\operatorname{sign}(e) \omega^{1 / 2}\right)\left[(e-1)^{2}+f^{2}\right]-2 f^{2}\right]} .
$$

The maximum value of $|B|^{2}$ is

$$
B_{p v}=\frac{e(1+\omega)(\omega-1)^{2} f^{2}}{2 \omega^{2}\left[\left(e+\operatorname{sign}(e) \omega^{1 / 2}\right)\left[(e-1)^{2}+f^{2}\right]-2 f^{2}\right]} .
$$




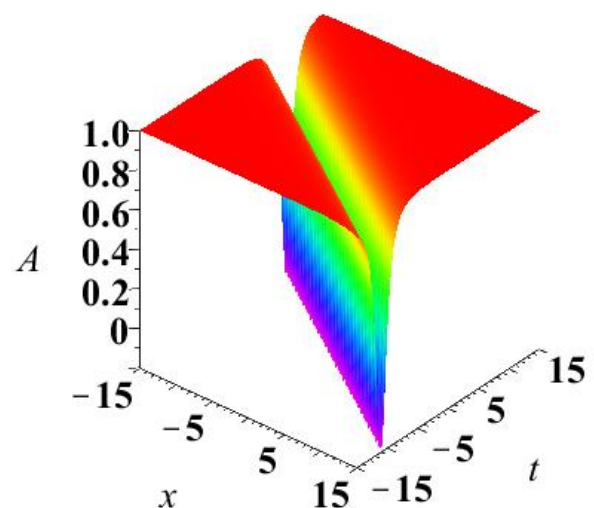

(a)

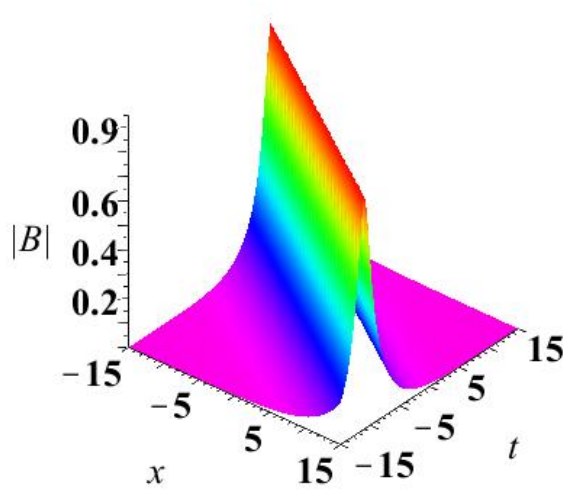

(c)

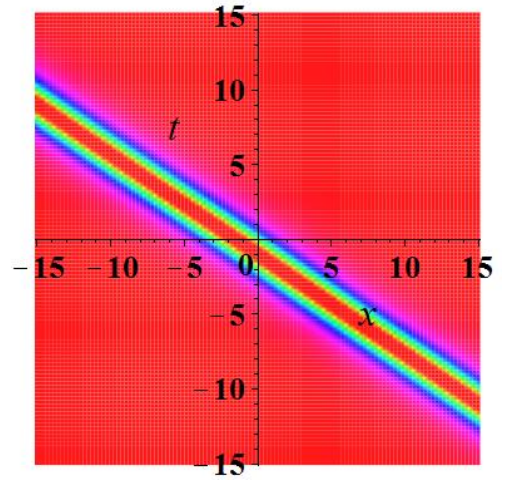

(b)

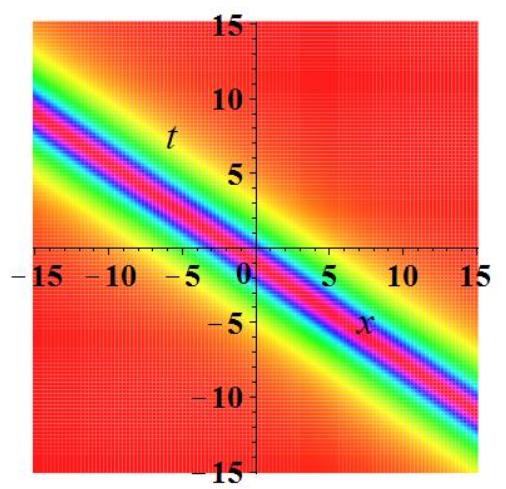

(d)

Fig. 1. (Color online)(a): Picture of $A(x, t)$; (b): Density plot of $A(x, t)$; (c): Picture of $|B(x, t)|$; (d): Density plot of $|B(x, t)|$; We can see that $A$ is a dark soliton and $|B|$ is a bright soliton. From the density plot, we can see that the trajectory of $A$ and $|B|$ is along the same line.

To give us a clear understanding of the solutions, we exhibit the dynamics of solution $A$ and $|B|$ by plotting the picture. Choosing parameters $c_{1}=0, c_{2}=1$ and $e=f=1$, we plot the explicit picture by Maple (Fig. 1). It is seen that the solution $A$ is a dark soliton, and $|B|$ is a bright soliton.

If we choose the parameters $c_{1} \neq 0$ and $c_{2}=0, k=e+\mathrm{i} f$, $e f \neq 0$ and $e<0$, we can obtain the exact expression for $A$ and $B$ :

$$
\begin{aligned}
& A=1+\frac{\gamma}{\delta\left[e^{2}(\omega+1)^{2} e^{2 X_{1}}+\omega^{3} e^{-2 X_{1}}\right]+1} \\
& B=\frac{e f\left(1-\omega^{2}\right) e^{\mathrm{i} Y_{1}}}{\omega^{2}(1+e+\mathrm{i} f) e^{X_{1}}+e(1+\omega)(\omega+e+\mathrm{i} f) e^{-X_{1}}}
\end{aligned}
$$


where

$$
\begin{aligned}
X_{1} & =\frac{f}{2}\left(\frac{1}{\omega}-1\right)\left(x-\left(e+\frac{e}{\omega}\right) t+g_{1}\right), \quad \omega=e^{2}+f^{2}, \\
Y_{1} & =\frac{e}{2}\left(1+\frac{1}{\omega}\right)\left(x+\left(-\frac{e}{2}-\frac{e}{\omega}+\frac{\left(f^{2}+1\right)}{2 e}+\frac{2 e}{\omega+1}\right) t+g_{2}\right), \\
\gamma & =\frac{(\omega-1)^{2} f^{2}}{\omega\left[e\left((e+1)^{2}+f^{2}\right)+2 f^{2}\right]}, \quad g_{1}=\frac{2 \omega \ln \left|c_{2}\right|}{f(1-\omega)} \\
\delta & =\frac{(e+1)^{2}+f^{2}}{2 e \omega(\omega+1)\left[e\left((e+1)^{2}+f^{2}\right)+2 f^{2}\right]}, \quad g_{2}=\frac{2 \arg \left(c_{2}\right) \omega}{e(1+\omega)}
\end{aligned}
$$

The trajectories of $A$ and $|B|^{2}$ are along the line $X_{1}+\ln (|e|(1+\omega))-\frac{3}{2} \ln (\omega)=0$. The cavity depth for $A$ is

$$
A_{c d}=\frac{-(\omega-1)^{2} f^{2}}{\omega\left[\left(e+\operatorname{sign}(e) \omega^{1 / 2}\right)\left[(e+1)^{2}+f^{2}\right]+2 f^{2}\right]} .
$$

The maximum value of $|B|^{2}$ is

$$
B_{p v}=\frac{e(1+\omega)(\omega-1)^{2} f^{2}}{2 \omega^{2}\left[\left(e+\operatorname{sign}(e) \omega^{1 / 2}\right)\left[(e+1)^{2}+f^{2}\right]+2 f^{2}\right]} .
$$

\subsubsection{Bright-Bright pair}

If we choose the parameters $c_{1}=0$ and $c_{2} \neq 0, k=e+\mathrm{i} f, e f \neq 0$ and $e<0$, we can obtain the exact expression for $A$ and $B$ (3.3). If we choose the parameters $c_{1} \neq 0$ and $c_{2}=0, k=e+\mathrm{i}$ f, ef $\neq 0$ and $e>0$, we can obtain the exact expression for $A$ and $B$ (3.4). Because the properties are similar with the Bright-Dark pair, we ignore it in this subsection.

To give us a clear understanding of the solutions, we exhibit the dynamics of solution $A$ and $|B|$ by plotting the picture. Choosing parameters $c_{1}=1, c_{2}=0$ and $e=f=1$, we can plot the picture of $A(x, t)$ and $|B(x, t)|$ (Fig. 2) by Maple.

\subsubsection{New type soliton pair}

Before giving this kind of soliton solution, we choose the parameters $c_{2}=1, c_{3}=0$ and divide $\left|x_{1}\right\rangle$ by the factor $\exp \left[\frac{i}{6}\left(k-k^{-1}\right)^{2} t-\frac{i}{4}\left(k-k^{-1}\right)^{2} t\right]$, then components of the vector solution $\left|x_{1}\right\rangle$ are

$$
\begin{aligned}
\phi_{1} & =c_{1} \exp \left[\frac{\mathrm{i}}{2}\left(k+k^{-1}\right) x\right]+c_{2} \exp \left[-\frac{\mathrm{i}}{2}\left(k+k^{-1}\right) x\right], \\
\phi_{2} & =0, \\
\phi_{3} & =c_{1} k^{-1} \exp \left[\frac{\mathrm{i}}{2}\left(k+k^{-1}\right) x\right]-c_{2} k \exp \left[-\frac{\mathrm{i}}{2}\left(k+k^{-1}\right) x\right] .
\end{aligned}
$$

Then we can obtain the trivial solutions by formulas (2.6)

$$
\begin{aligned}
& A=-\frac{2}{\alpha^{2}-\beta^{2}}\left[\beta\left(\phi_{1} \phi_{3}^{*}-\phi_{3} \phi_{1}^{*}\right)+\alpha\left(\left|\phi_{3}\right|^{2}-\left|\phi_{1}\right|^{2}\right)\right], \\
& B=0
\end{aligned}
$$




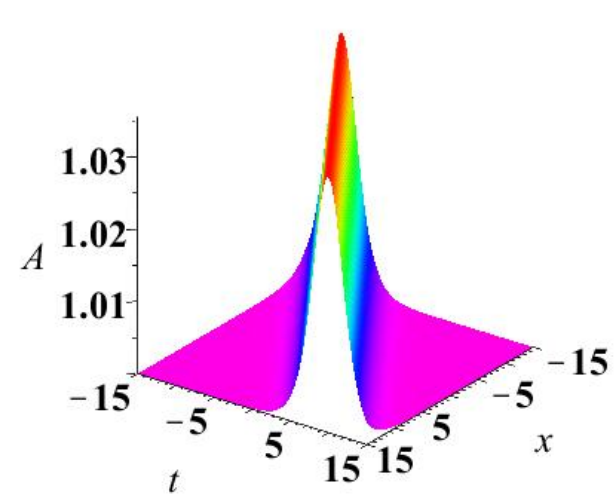

(a)

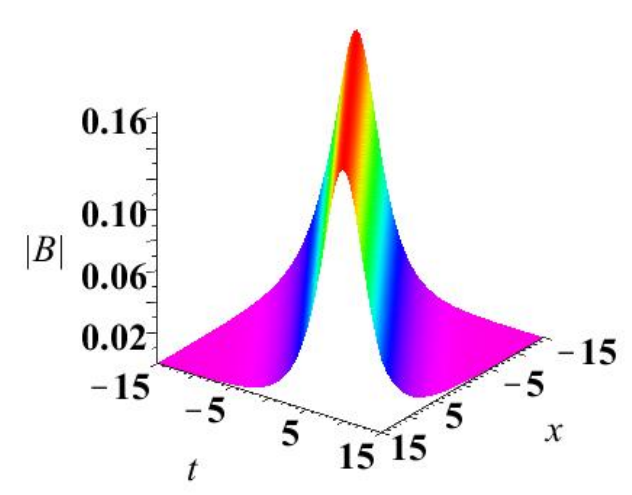

(c)

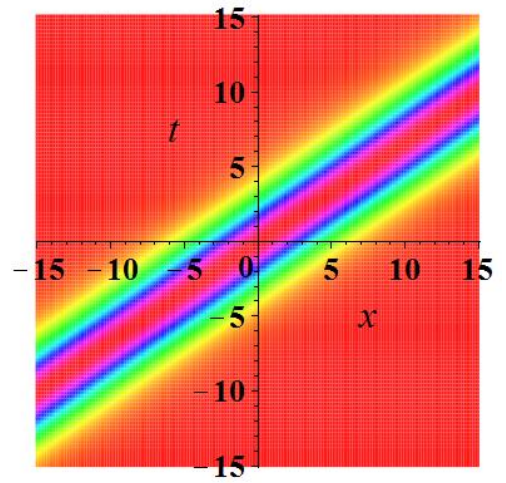

(b)

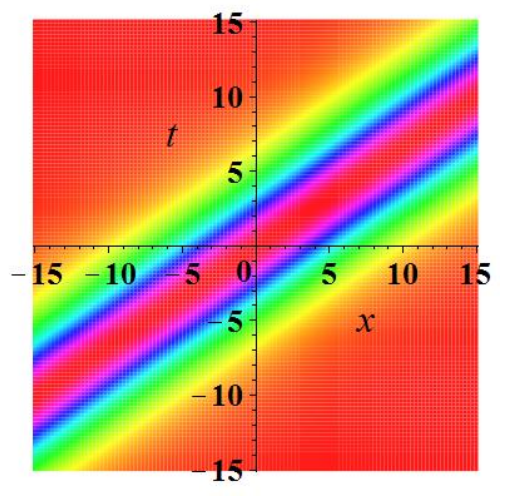

(d)

Fig. 2. (Color online) (a): Picture of $A(x, t)$; (b): Density picture of $A(x, t)$;(c): Picture of $|B(x, t)|$; (d): Density picture of $|B(x, t)|$; We can see that they are both bright soliton. However, the amplitude of bright-bright pair is lower than the bright-dark pair.

where

$$
\alpha=\frac{\operatorname{Re}\left(\phi_{1}^{*} \phi_{3}\right)}{\operatorname{Re}^{2}\left(\lambda_{1}\right)}, \quad \beta=\frac{\left|\phi_{1}\right|^{2}+\left|\phi_{3}\right|^{2}}{2 \operatorname{iIm}^{2}\left(\lambda_{1}\right)}
$$

We can see that the solution $A(x, t)$ is independent with $t$. On the other hand, we know that any solution $A(x)$ and $B=0$ satisfy the equation (1.2). Thus the solution (3.6) is a trivial solution.

If we choose the parameters $c_{1} c_{2} \neq 0$ and $c_{3}=1$, then we could obtain the new type soliton solution. In mathematical view, this kind of solution is nothing but the nonlinear superposition for different type solutions. For convenience, we merely consider the parameters $e>0$. 
We analyse the asymptotic behavior of new type soliton solution. If $e^{2}+f^{2}>1$, ef $>0$ and $t>0$, then the vector solution (3.2), as $t \rightarrow+\infty$

$$
\left|x_{1}\right\rangle \rightarrow\left[\begin{array}{c}
\exp \left[-\frac{\mathrm{i}}{4}\left(k-k^{-1}\right)^{2} t\right]\left(c_{1} \exp \left[\frac{\mathrm{i}}{2}\left(k+k^{-1}\right) x\right]+c_{2} \exp \left[-\frac{\mathrm{i}}{2}\left(k+k^{-1}\right) x\right]\right) \\
0 \\
\exp \left[-\frac{\mathrm{i}}{4}\left(k-k^{-1}\right)^{2} t\right]\left(c_{1} k^{-1} \exp \left[\frac{\mathrm{i}}{2}\left(k+k^{-1}\right) x\right]-c_{2} k \exp \left[-\frac{\mathrm{i}}{2}\left(k+k^{-1}\right) x\right]\right)
\end{array}\right]
$$

It follows that we have solution (3.6). As $t \rightarrow-\infty$, without generality we suppose $f>0$, and $x \rightarrow$ $-\infty$, then the vector solution $(3.2) \wedge$

$$
\left|x_{1}\right\rangle \rightarrow\left[\begin{array}{c}
c_{1} \exp \left[\frac{\mathrm{i}}{2}\left(k+k^{-1}\right) x-\frac{\mathrm{i}}{4}\left(k-k^{-1}\right)^{2} t\right] \\
1 \\
c_{1} k^{-1} \exp \left[\frac{\mathrm{i}}{2}\left(k+k^{-1}\right) x-\frac{\mathrm{i}}{4}\left(k-k^{-1}\right)^{2} t\right]
\end{array}\right] .
$$

It follows that we have solution (3.4). As $t \rightarrow-\infty, f>0$, and $x \rightarrow+\infty$, then the vector solution $(3.2) \wedge$

$$
\left|x_{1}\right\rangle \rightarrow\left[\begin{array}{c}
c_{2} \exp \left[-\frac{\mathrm{i}}{2}\left(k+k^{-1}\right) x-\frac{\mathrm{i}}{4}\left(k-k^{-1}\right)^{2} t\right] \\
1 \\
-c_{2} k \exp \left[-\frac{\mathrm{i}}{2}\left(k+k^{-1}\right) x-\frac{\mathrm{i}}{4}\left(k-k^{-1}\right)^{2} t\right]
\end{array}\right]
$$

It follows that we have solution (3.3).

To give us a clear understanding of the solutions, we exhibit the dynamics of solution $A$ and $|B|$ by plotting the picture. Choosing parameters $c_{1}=c_{2}=1$ and $e=f=1$, we can obtain the pictures of $A(x, t)$ and $|B(x, t)|$ and some other pictures for $A(x, t)$ (Fig. 3). Similar phenomena are reported in reference [3]. Although the spectral problem for this model (1.2) is deep reduction for the WRI model [3], this model (1.2) is second flow for this spectral problem with deep reduction and WRI model is the first nontrivial flow without deep reduction. Thus the solutions we obtained can not be reduced from the solution of previous research papers $[3,4]$.

\subsection{The Case II}

In this case, taking parameters $a=d=0$, we merely take parameters $c=1$ by scalings. As above subsection, we make a substitution $\lambda_{1}=\frac{1}{2}\left(k-\frac{2}{k}\right) \notin \mathbb{R} \cup i \mathbb{R}$. Then solutions can be represented as

$$
\begin{aligned}
A & =-\frac{2}{\alpha^{2}-\beta^{2}}\left[\beta\left(\phi_{1} \phi_{3}^{*}-\phi_{3} \phi_{1}^{*}\right)+\alpha\left(\left|\phi_{3}\right|^{2}-\left|\phi_{1}\right|^{2}\right)\right], \\
B & =\left(1-\mathrm{i} \frac{\left(\phi_{3}-\phi_{1}\right) \phi_{2}^{*}}{\alpha+\beta}\right) e^{2 \mathrm{i} t},
\end{aligned}
$$




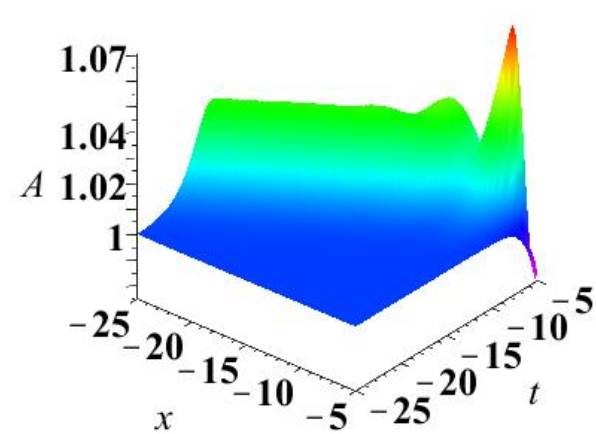

(a)

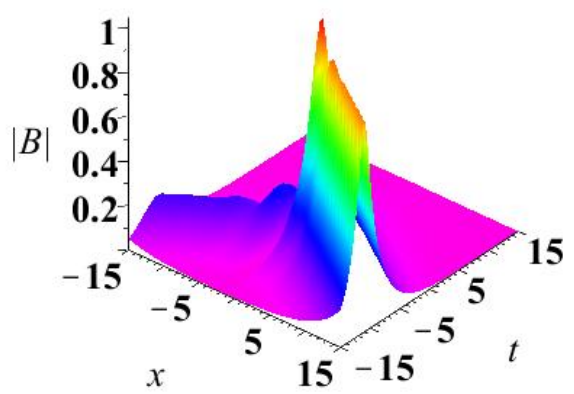

(c)

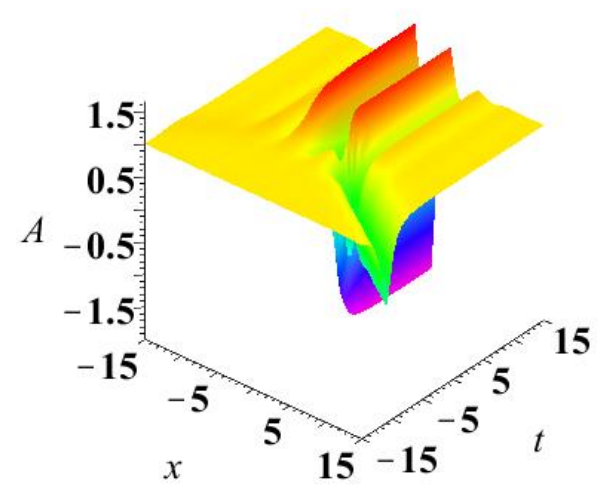

(b)

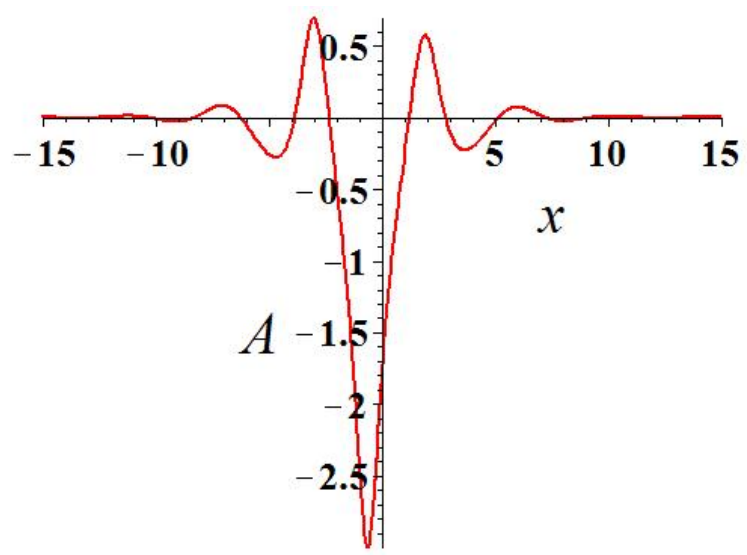

(d)

Fig. 3. (Color online) (a),(b) Picture of $A$; (c) Picture of $|B|$; (d), the picture of $A$, when $t$ is enough big $\left(t=10^{4}\right)$. It is very interesting that we can obtain a new kind of solution by nonlinear superposition of bright-dark pair, bright-bright pair and the trivial case.

where

$$
\begin{aligned}
\phi_{1} & =\frac{2 \mathrm{i} k}{k^{2}-2}-\frac{\mathrm{i} k}{2} c_{1} \exp \left(X_{1}\right)+\frac{\mathrm{i}}{k} c_{2} \exp \left(X_{2}\right), \\
\phi_{2} & =1+c_{1} \exp \left(X_{1}\right)+c_{2} \exp \left(X_{2}\right), \\
\phi_{3} & =-\frac{2 \mathrm{i} k}{k^{2}-2}-\frac{\mathrm{i}}{k} c_{1} \exp \left(X_{1}\right)+\frac{\mathrm{i} k}{2} c_{2} \exp \left(X_{2}\right), \\
\alpha & =\frac{\left|\phi_{2}\right|^{2}+2 \operatorname{Re}\left(\phi_{1}^{*} \phi_{3}\right)}{2 \operatorname{Re}^{2}\left(\lambda_{1}\right)}, \quad \beta=\frac{\left|\phi_{1}\right|^{2}+\left|\phi_{2}\right|^{2}+\left|\phi_{3}\right|^{2}}{2 \operatorname{im}^{2}\left(\lambda_{1}\right)},
\end{aligned}
$$

and

$$
\begin{aligned}
& X_{1}=\mathrm{i}\left(k / 2+k^{-1}\right)\left[x+\left(k / 2+k^{-1}\right) t\right], \\
& X_{2}=\mathrm{i}\left(k / 2+k^{-1}\right)\left[-x+\left(k / 2+k^{-1}\right) t\right],
\end{aligned}
$$




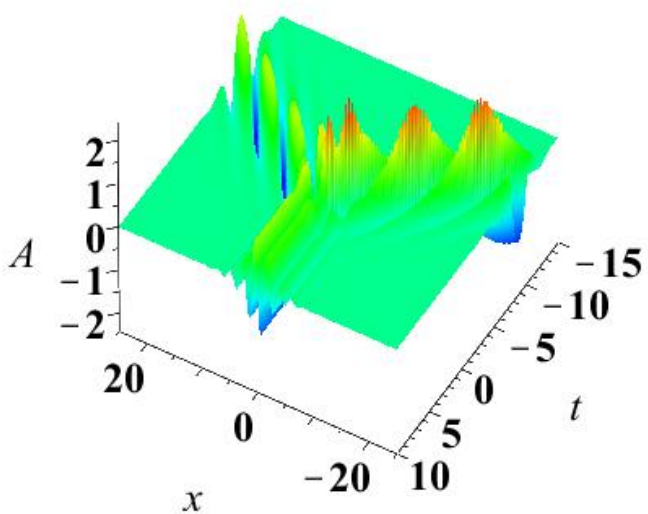

(a)

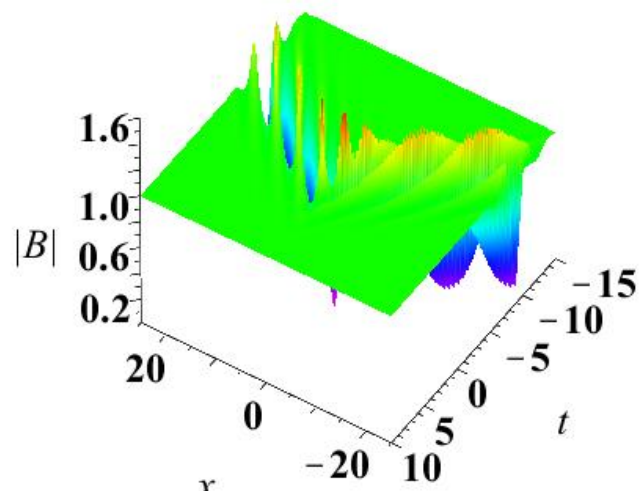

(c)

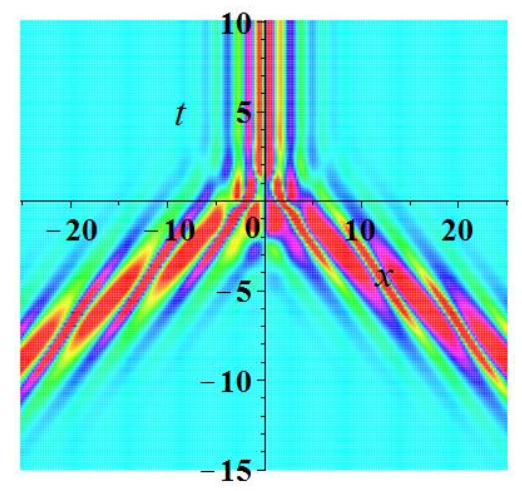

(b)

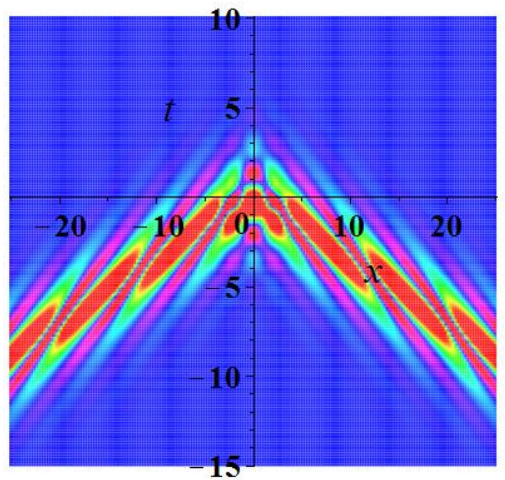

(d)

Fig. 4. (Color online) (a): Picture of $A(x, t)$; (b): Density plot of $A(x, t)$; (c): Picture of $|B(x, t)|$; (d): Density plot of $|B(x, t)|$; We can see the breather solutions possess the similar structure with Fig. 3 .

$c_{1}$ and $c_{2}$ are complex constants. Similar as above subsection, we can obtain three different kinds of solution.

Choosing parameters $c_{1}=c_{2}=1$ and $k=2+\mathrm{i}$, we can obtain the picture of $A(x, t)$ and $|B(x, t)|$ (Fig. 4) by Maple. It is seen that two breathers get together into a stationary soliton from the picture and density plot of $A$, and two breathers annihilate from the picture and density plot of $|B|$.

\subsection{Case III}

In this subsection, we consider the general case. To solve the periodic coefficient ODE, we use the gauge transformation. We use the gauge transformation

$$
g_{0}=\operatorname{diag}(\exp (-\mathrm{i} \chi / 3), \exp (2 \mathrm{i} \chi / 3), \exp (-\mathrm{i} \chi / 3))
$$


where $\chi=a x+\left(d^{2}+2 c^{2}-a^{2}\right) t$. Then the linear system (1.3) becames

$$
\begin{aligned}
& \Psi_{x}=U_{0} \Psi, \\
& \Psi_{t}=\left(\mathrm{i} U_{0}^{2}-\frac{2 a}{3} U_{0}+\frac{2 \mathrm{i}}{9}\left(3 \lambda_{1}^{2}+3 d^{2}+a^{2}+6 c^{2}\right)\right) \Psi,
\end{aligned}
$$

where

$$
U_{0}=\left[\begin{array}{ccc}
\mathrm{i}\left(\lambda_{1}-a / 3\right) & c & \mathrm{i} d \\
-c & 2 \mathrm{i} a / 3 & -c \\
\mathrm{i} d & c & -\mathrm{i}\left(\lambda_{1}+a / 3\right)
\end{array}\right]
$$

The next task is diagonalization of matrix $U_{0}$. Firstly, we have the characteristic equation

$$
\xi^{3}+\left(\lambda_{1}^{2}+d^{2}+2 c^{2}+a^{2} / 3\right) \xi+\mathrm{i}\left(-2 a \lambda_{1}^{2} / 3+2 a^{3} / 27+2 c^{2} d+2 a c^{2} / 3-2 a d^{2} / 3\right)=0 .
$$

Suppose above cubic equation has three different solution $\xi_{1}, \xi_{2}, \xi_{3}$ respectively. Then the solution of equations (3.8) can be represented as

$$
\begin{aligned}
& \phi_{1}=A_{1} \exp \left(X_{1}\right)+A_{2} c_{1} \exp \left(X_{2}\right)+A_{3} c_{2} \exp \left(X_{3}\right), \\
& \phi_{2}=B_{1} \exp \left(X_{1}\right)+B_{2} c_{1} \exp \left(X_{2}\right)+B_{3} c_{2} \exp \left(X_{3}\right), \\
& \phi_{3}=\exp \left(X_{1}\right)+c_{1} \exp \left(X_{2}\right)+c_{2} \exp \left(X_{3}\right),
\end{aligned}
$$

where

$$
\begin{aligned}
A_{i} & =\frac{-3\left(-3 c^{2}+2 a d+3 \mathrm{i} d \xi_{i}\right)}{-2 a^{2}+3 \mathrm{i} a \xi_{i}-9 \xi_{i}^{2}+6 a \lambda_{1}+9 \mathrm{i} \lambda_{1} \xi_{i}-9 c^{2}}, \\
B_{i} & =\frac{3 c\left(3 \mathrm{i} d+\mathrm{i} a+3 \xi_{i}-3 \mathrm{i} \lambda_{1}\right)}{-2 a^{2}+3 \mathrm{i} a \xi_{i}-9 \xi_{i}^{2}+6 a \lambda_{1}+9 \mathrm{i} \lambda_{1} \xi_{i}-9 c^{2}}, \\
X_{i} & =\xi_{i} x+\left(\mathrm{i} \xi_{i}^{2}-\frac{2 a}{3} \xi_{i}+\frac{2 \mathrm{i}}{9}\left(3 \lambda_{1}^{2}+3 d^{2}+a^{2}+6 c^{2}\right)\right) t .
\end{aligned}
$$

Then solution can be represented as

$$
\begin{aligned}
& A=d-\frac{2}{\alpha^{2}-\beta^{2}}\left[\beta\left(\phi_{1} \phi_{3}^{*}-\phi_{3} \phi_{1}^{*}\right)+\alpha\left(\left|\phi_{3}\right|^{2}-\left|\phi_{1}\right|^{2}\right)\right], \\
& B=\left(c-\mathrm{i} \frac{\left(\phi_{3}-\phi_{1}\right) \phi_{2}^{*}}{\alpha+\beta}\right) \exp \left[\mathrm{i}\left(a x+\left(d^{2}+2 c^{2}-a^{2}\right) t\right)\right],
\end{aligned}
$$

where

$$
\alpha=\frac{\left|\phi_{2}\right|^{2}+2 \operatorname{Re}\left(\phi_{1}^{*} \phi_{3}\right)}{2 \operatorname{Re}^{2}\left(\lambda_{1}\right)}, \quad \beta=\frac{\left|\phi_{1}\right|^{2}+\left|\phi_{2}\right|^{2}+\left|\phi_{3}\right|^{2}}{2 \operatorname{iIm}^{2}\left(\lambda_{1}\right)},
$$

and $\lambda_{1} \notin \mathbb{R} \cup \mathrm{i} \mathbb{R}$. 


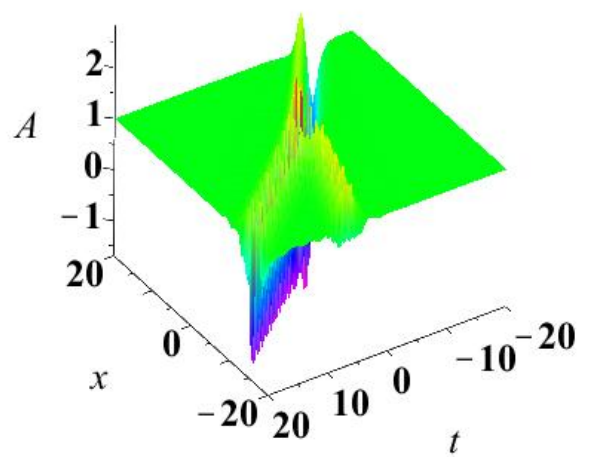

(a)

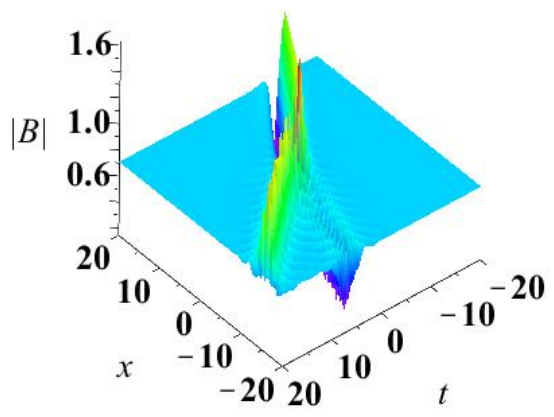

(c)

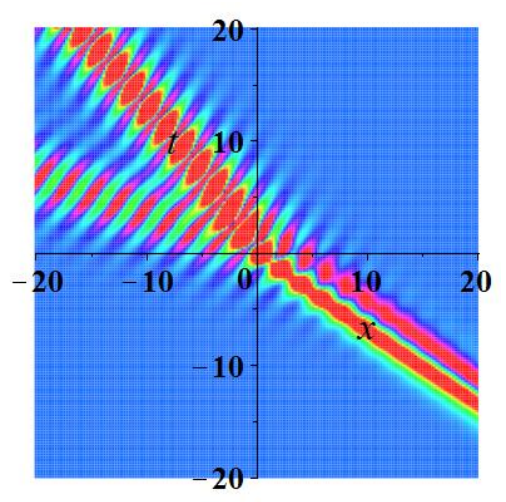

(b)

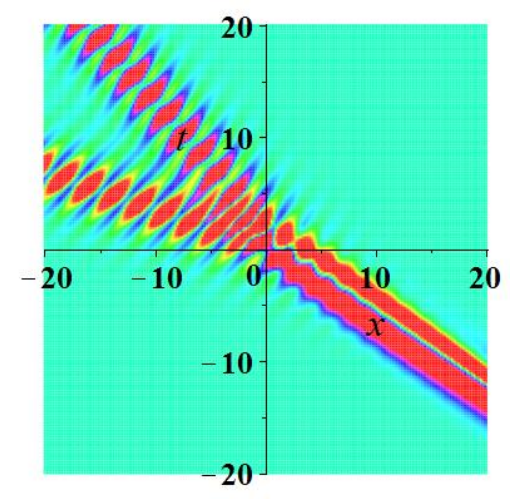

(d)

Fig. 5. (Color online) (a): Picture of $A(x, t)$; (b): Density picture of $A(x, t)$; (c): Picture of $|B(x, t)|$; (d): Density picture of $|B(x, t)|$; This kind of solution is different from Fig. 4. When $t$ is enough big, $A$ is not stationary and $|B|$ is no longer vanishing.

Choosing parameters

$$
a=0, \quad d=1, \quad c=2^{-1 / 2}, \quad \lambda_{1}=(1+3 \mathrm{i}) / 4,
$$

then we can numerically solve the cubic equation (3.10) by Maple

$$
\begin{aligned}
& \xi_{1} \approx-0.3413199783-0.5734343555 \mathrm{i}, \\
& \xi_{2} \approx-0.1099240564+1.478137206 \mathrm{i}, \\
& \xi_{3} \approx 0.4512440348-0.9047028502 \mathrm{i} .
\end{aligned}
$$

Owning to the functions $A(x, t)$ and $B(x, t)$ (3.11) with respect to the parameters $\xi_{i}, i=1,2,3$, are continuous, the errors between the numeric solution and exact solution are enough small. Substituting the parameters (3.12) and (3.13) into formulas (3.11), and putting them into computer, we can obtain the picture and density plot of solutions $A(x, t)$ and $|B(x, t)|$ (Fig. 5). It is seen that the soliton $|B|$ does not annihilate. 


\section{Conclusions and discussions}

In this work, we analyse the exact solution for long wave-short wave model (1.2) with non-vanishing background. However, we can not obtain the rational solution for this model (1.2), since the deep reduction of Darboux transformation.

\section{Acknowledgments}

This work is supported by National Natural Science Foundation of China 11271052.

\section{References}

[1] D. Bian, B. Guo and L. Ling, Landau-Lifshitz equation: High-order soliton and long time asymptotics. Subbmitted

[2] D. J. Benney, A general theory for interactions between short and long waves, Stud. Appl. Math. 56 (1977) 81-94.

[3] F. Calogero, A. Degasperis Novel solution of the system describing the resonant interaction of three waves, Physica D 200 (2005) 242-256.

[4] M. Conforti, F. Baronio and A. Degasperis, Modulational instability of dark solitons in three wave resonant interaction, Physica D 240 (2011) 1362-1369.

[5] J. Ciéliński, Algebraic construction of the Darboux matrix revisited. J. Phys. A: Math. Theor. 42 (2009) 404003.

[6] A. Degasperis, M. Conforti, F. Baronio, S. Wabnitz and S. Lombardo, The Three-Wave Resonant Interaction Equations: Spectral and Numerical Methods, Lett. Math. Phys. 96 (2011) 367-403.

[7] C.H. Gu, H.S. Hu, and Z.X. Zhou, Darboux Transformation in Soliton Theory, and its Geometric Applications, (Shanghai Science and Technology Publishers, Shanghai 2005)

[8] B. Guo and L. Chen, Orbital Stability of Solitary Waves of the Long Wave-Short Wave Resonance Equations, Math. Meth. Appl. Sci. 21 (1998) 883-894.

[9] B. Guo, L. Ling, and Q. P. Liu, Nonlinear Schrödinger equation: Generalized Darboux transformation and rogue wave solutions. Phys. Rev. E 85 (2012) 026607.

[10] B. Guo, L. Ling and Q. P. Liu, High-Order Solutions and Generalized Darboux Transformations of Derivative Nonlinear Schrödinger Equations, Stud. Appl. Math. 130(2013) 317-344.

[11] B. Guo, L. Ling, Rogue Wave, Breathers and Bright-Dark-Rogue Solutions for the Coupled Schrodinger Equations, Chin. Phys. Lett. 28 (2011) 110202.

[12] B. Guo and X. Pan, $N$-soliton solutions for a class of the system of $L S$ nonlinear wave interaction, Chin. Phys. Lett. 7 (1990) 241-244.

[13] D.J. Kaup, The three-wave interactionta nondispersive phenomenon, Stud. Appl. Math. 55 (1976) 9-44.

[14] L. Ling, and Q. P. Liu, A long waves-short waves model: Darboux transformation and soliton solutions, J. Math. Phys. 52 (2011) 053513.

[15] Y. C. Ma, The complete solution of the long-wave-short-wave resonance equations, Stud. Appl. Math. 59 (1978) 201-221.

[16] V. Matveev and M. Salle, Darboux transformation and solitons, Springer-Verlag, Berlin (1991)

[17] A. C. Newell, Long waves-short waves; a solvable model, SIAM J. Appl. Math. 35 (1978) 650-664.

[18] C.-L. Terng and K. Uhlenbeck, Bäcklund transformations and loop group actions, Comm. Pure Appl. Math. 53 (2000) 1-75.

[19] M. Tsutsumi and S. Hatano, Well posedness of the cauchy problem for the long wave-short wave resonance equations, Nonlinear Anal. T.M.A. 22 (1994) 155-171.

[20] V.E. Zakharov, and S.V. Manakov, Resonance interaction of wave packets, Sov. Phys. JEPT Lett. 18 (1973) 243-245. 